\title{
Caution: tripping hazards
}

\section{N. Hutchins $\dagger$}

Walter Bassett Aerodynamics Laboratory, Department of Mechanical Engineering, University of Melbourne, Victoria 3010, Australia

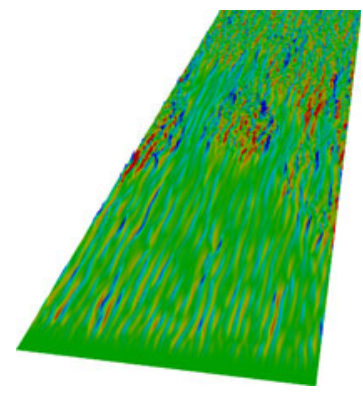

A turbulent boundary layer formed over an external surface is spatially evolving. The flow develops from an initial set of conditions and accordingly the state of the layer at some downstream location owes a debt to its upstream history. Schlatter \& Örlü (J. Fluid Mech., this issue, vol. 710, 2012, pp. 5-34) demonstrate succinctly this sensitivity to upstream history, finding that relatively minor modifications to the trip parameters can produce non-canonical development up to surprisingly high Reynolds numbers. This interesting study will serve as a cautionary note to experimentalists and numericists while providing a plausible explanation for some of the disparity noted among previously published results.

Key words: transition to turbulence, turbulence simulation, turbulent boundary layers

\section{Introduction}

It is probably safe to assume that most scientists will favour evolution over creationism, the former being well-based in accepted scientific rationale. The recent paper by Schlatter \& Örlü (2012, this issue) reminds us that the same rigour should be applied to our consideration of turbulent boundary layers. Turbulent boundary layers are the thin regions of chaotic highly sheared flow dragged along by vehicles as they move through a fluid (or alternatively that form adjacent to a boundary as a fluid flows past a solid object). These layers are of critical importance to the efficiency and performance of a number of key engineering and natural systems. Regardless of the precise scenario, the turbulent boundary layer is always considered to develop from some starting or inception point. On aircraft or ships, this inception point typically occurs just downstream of the leading edge of the vehicle, and for laboratory or numerical experiments it is often necessary to artificially prompt transition to turbulence using some form of obstruction, surface roughness or energy input that is typically referred to as a 'trip'. The work of Schlatter \& Örlü (2012) reminds us that the layer retains some memory of these initial tripping conditions as it develops downstream of this inception point. In this sense the turbulent boundary layer truly does evolve from an initial set of conditions, and information pertaining to these initial conditions is retained in the layer for some distance downstream of its inception.

In many respects, this news is not new, perhaps just unwelcome. Indeed, the need for caution in judiciously selecting trip parameters has been expressed repeatedly in the literature going back over half a century (Klebanoff \& Diehl 1951; Coles 1969; Erm \& Joubert 1991; Chauhan, Monkewitz \& Nagib 2009). However, as

$\dagger$ Email address for correspondence: nhu@unimelb.edu.au 
a community, it seems that we require constant reminding. It is easy to understand why such news could be unwelcome, raising as it does questions on the commutability of measurements between different facilities and even challenging that most cherished of fluid mechanics constructs: Reynolds number similarity. However, at a time when computational power and the will of the community has reached the point where developing boundary layers are now being directly simulated over very long numerical boxes, the work of Schlatter \& Örlü (2012) serves as a timely reminder that boundary layers evolve, the tripping process is formative, and therefore these flows retain a memory of their initiation. Reynolds number similarity and a truly canonical flow can only be attained once this memory is drowned out by the march of turbulence towards some quasi-equilibrium state.

Schlatter \& Örlü (2012) precisely demonstrate that residual artifacts from tripping can potentially corrupt results from direct numerical simulations (DNS) of developing turbulent boundary layers. This is important since at present it remains almost impossible for researchers to conduct well-controlled numerical or laboratory experiments on turbulent boundary layers at conditions that precisely match those that would exist in most real-life scenarios. Accordingly, results from low-Reynoldsnumber experiments must be translated (or extrapolated) up to real-life conditions. This process is only justifiable if the low-Reynolds-number flows we study are considered canonical. The results of Schlatter \& Örlü (2012) clearly undermine this assumption. Compounding this issue, DNS results are often disproportionately weighted (over other experimental datasets) in studies investigating the Reynolds number development of certain attributes of turbulent boundary layers (perhaps owing to the general cleanliness of the data as compared to experimental results, and also to a view that DNS is largely incorruptible by technique). It is in this environment that the work of Schlatter \& Örlü (2012) should be judged. They cement the view that DNS of developing turbulent boundary layers should be viewed as a 'numerical experiment' with the resulting data 'subject to the same scrutiny as experimental data'. Wise experimentalists will also take note of this study, with low-Reynolds-number experiments likely to be every bit as susceptible to corruption from tripping artifacts.

\section{Overview}

The present work by Schlatter \& Örlü (2012) focuses on questions raised by their previous paper (Schlatter \& Örlü 2010), which through careful comparison of existing DNS data from developing turbulent boundary layers, concluded that such simulations, despite ostensibly simulating the same canonical flow, exhibit surprisingly large variations in basic integral quantities and mean profiles. Given the success of DNS in the turbulent channel flow case (a fully developed flow), the most likely candidate for the noted discrepancies with the turbulent boundary layer simulations was always most likely to lie somehow with the issue of development. Through a systematic variation of simulation parameters, Schlatter \& Örlü (2012) now demonstrate convincingly that most of the differences observed between the existing boundary layer DNS can be attributed to the different tripping conditions used in these simulations.

A total of eight different flows are simulated over very large domain sizes, of which four are visualized in figure 1. Tripping is achieved in these simulations via a random volume force (acting in the wall-normal direction), with the amplitude, frequency and location $\left(R e_{\theta}\right)$ of the tripping varied across the simulations. By analysing the development of various parameters downstream of the trip (shape factor, momentum and displacement thickness, skin friction, profile shape and various fluctuating quantities), the authors unequivocally demonstrate that the choice of 


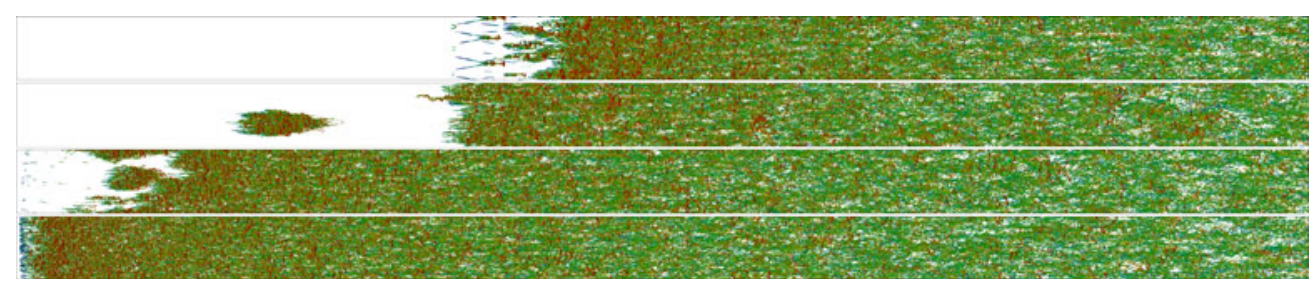

FIGURE 1. A selection of top views of developing boundary layers under different tripping conditions from simulations of Schlatter \& Örlü (2012) showing the corresponding streamwise extent of the different DNS domains. The streamwise development of vortical structures is identified by the $\lambda_{2}$ criterion. The end of the domain corresponds to different local Reynolds numbers; from top to bottom $R e_{\theta} \approx 2000,2100,2400,2500$.

tripping parameters has an obvious and lasting impact on the downstream development of the turbulent boundary layer. Provided that the tripping is initiated at low enough Reynolds numbers, they demonstrate that the developing boundary layer will exhibit sensitivity to these initial tripping conditions only up to $\operatorname{Re}_{\theta} \approx 2000$. While this may not unduly disturb experimentalists, who would typically be cautious of measuring so 'close' to the trip, this is actually approaching the outflow limit for many existing simulations (and thus a poor choice of tripping parameters could corrupt the entire numerical domain). The exception here are flows that have been tripped at higher Reynolds numbers (domains with higher inflow Reynolds numbers, or flows tripped further downstream of the inlet to the domain). In this case, the effects of the trip reported by Schlatter \& Örlü (2012) extend beyond the end of the measurement domain, and it is not clear precisely how much development length is required for the flow to forget its initial inception. Such scenarios have been used in previous simulations under the belief that this might offer a shortcut to higher-Reynolds-number simulation of turbulent boundary layers. This scenario could also arise in experiments, where the boundary layer coming onto the trip, has already attained a high Reynolds number (specifically, one could imagine this scenario occurring in situations where the boundary layer is developing over the bottom wall of a wind tunnel). Certainly this is an area that requires further investigation.

The good news here (from a 'glass half full' perspective) is that with increasing Reynolds numbers, all flows do seem to be converging to a common asymptote. Hence the sanctity of Reynolds-number similarity is apparently eventually obeyed. Regardless of how the turbulence is initially promoted, there is a settling length downstream of the trip beyond which upstream tripping history is forgotten and the flows settle down to a common canonical development. This is in contrast to the results of Castillo et al. (2001) who had questioned whether such an asymptotic state even exists. In their case, experimental data had seemed to suggest that flows with different initial conditions were converging to different asymptotic states. It should however be noted that the trip is but one of a host of upstream conditions that could potentially affect boundary layer evolution (including free-stream turbulence, pressure gradient, pressure gradient history and certain anomalies in the contraction).

\section{Future}

Schlatter \& Örlü (2012) will serve as a very useful reminder of the hazards resulting from 'poor judgement in the choice of tripping or thickening devices' (Coles 1969). 
While seemingly more immediately applicable to DNS practitioners, the results will hopefully also strike a resonant note for experimentalists. The authors' findings suggest that the tripping artifacts are absent from the flow by $R e_{\theta} \approx 2000$ provided that transition is initiated: (i) within the boundary layer and (ii) at low enough Reynolds number; and that (iii) over- or under-stimulation of the flow is avoided. One suspects that the devil here is in the detail of those three provisos. While proviso (i) would typically be met for most high-quality research tunnels with low free-stream intensities, provisos (ii) and (iii) are much less guaranteed. It is possible that boundary layers developing over the bottom wall of wind tunnels could potentially have quite high inflow Reynolds numbers onto the trip. Additionally, the range of tripping investigated by Schlatter \& Örlü (2012) was relatively restricted, and it is not immediately obvious that this would cover the entire parameter space that one could encounter experimentally. Particularly worrying in this regard is the manner in which the Reynolds number range is investigated in wind-tunnel experiments. To get higher Reynolds numbers experimentally, one typically either moves the measurement station further downstream of the trip, or increases the free-stream velocity. In the former case, the tripping parameters would remain constant. However, raising the free-stream velocity of the facility is likely to raise the amplitude of the tripping input, risking over-stimulation of the flow. Certainly, further work in this area would be warranted.

The conclusions of Schlatter \& Örlü (2012) will rightly evoke some concern over whether the flows we study are truly canonical. However, we should not lose sight of the wider implications of this research. As pointed out a decade ago by Castillo \& Johansson (2002), the fact that upstream conditions can have so profound an influence on downstream development might imply that flow control due to upstream manipulations could provide a viable avenue of research for turbulent boundary layer control. In addition, the study will force us to ponder the state of boundary layers that exist in real-life applications, where the trip forcing could conceivably be far from the optimal configurations suggested by Schlatter \& Örlü (2012).

\section{References}

Castillo, L. \& Johansson, T. G. 2002 The effects of the upstream conditions on a low Reynolds number turbulent boundary layer with zero pressure gradient. J. Turbul. 3 (N31), $1-19$.

Castillo, L., Seo, J., Walker, D., Johansson, G., Hangan, H. \& Blissitt, M. 2001 Turbulent boundary layers at very high Reynolds number and its relation to the initial conditions. AIAA Paper 2001-2913.

Chauhan, K. A., Monkewitz, P. A. \& Nagib, H. M. 2009 Criteria for assessing experiments in zero pressure gradient boundary layers. Fluid Dyn. Res. 41 (2), 021404.

Coles, D. E. 1969 Turbulent boundary layers in pressure gradients. Tech. Rep. RM-6142-PR, USAF. The RAND corporation.

ERM, L. P. \& JouberT, P. N. 1991 Low-Reynolds-number turbulent boundary layers. J. Fluid Mech. 230, 1-44.

Klebanoff, P. S. \& Diehl, Z. W. 1951 Some features of artificially thickened fully developed turbulent boundary layers with zero pressure gradient. NACA Tech. Rep. TN 2475.

SCHLATTER, P. \& ÖRLÜ, R. 2010 Assessment of direct numerical simulation data of turbulent boundary layers. J. Fluid Mech. 659, 116-126.

SChlatter, P. \& ÖRlü, R. 2012 Turbulent boundary layers at moderate Reynolds numbers: inflow length and tripping effects. J. Fluid Mech. 710, 5-34. 